\title{
Ship Detection Based on Information Theory and Segmentation from Synthetic Aperture Radar (SAR) Images
}

\author{
Somaya Jafari, Anisha M Lal, Jagalingam P
}

\begin{abstract}
Ship detection is a procedure which asserts in fields such as ocean and sea management, vessel detection, marine superintendence, and rein, and also can be applied to exclude extralegal actions. Remote sensing can be utilized as a potential tool for zonular and universal monitoring to attain the forenamed goals. Among the radar images, the precious datum from Synthetic Aperture Radar (SAR) is playing a serious duty in remote sensing. Howsoever, vessel detecting in heterogeneous and strong clutter is still a question in this regard. The letter points to a ship detection scheme for SAR images exploiting a segmentation-based morphological operation using entropy. In the presented scheme, the morphological operations are adopted to intercept the background and foreground in the satellite images. The method was implemented and tested on the homogenous, heterogeneous and strong clutter SAR images and the results are promising and showing that the proposed method can improve the vessel detection from homogenous and heterogeneous and strong clutter satellite images.
\end{abstract}

Keywords: Synthetic Aperture Radar, Ship Detection, morphological operations, entropy

\section{INTRODUCTION}

Maritime transportation have a rich affect in the country's economy. It is also required to rein the huge waters in other fields suchlike shipbuilding, safety [1] transportation mystery, service ministration, marine observation of maritime terrorism and prevention of unallowable activities [2] as well as search and rescue operations. Remote sensing can be employed as a possible gadget for zonal and planetary monitoring to attain the mentioned goals. Ship discovery in oceans and ports has been studied by many researchists using SAR data. Segmentation is a fundamental preparation phase needed to prospect the sea region for combat benefit. The primary aim is to segment the huge image regions in small parts to aid affirm the sea area differentiation and the

Manuscript received on February 10, 2020.

Revised Manuscript received on February 20, 2020.

Manuscript published on March 30, 2020.

* Correspondence Author

Somaya Jafari*, School of Computer Science and Engineering, Vellore Institute of Technology, Vellore, India. Email: smayajafari@gmail.com

Anisha M. Lal, School of Computer Science and Engineering, Vellore Institute of Technology, Vellore, India. Email: anishamlal@vit.ac.in

Jagalingam P, School of Computer Science and Engineering, Vellore Institute of Technology, Vellore, India. Email: jagalingam.p@vit.ac.in

(C) The Authors. Published by Blue Eyes Intelligence Engineering and Sciences Publication (BEIESP). This is an open access article under the CC BY-NC-ND license (http://creativecommons.org/licenses/by-nc-nd/4.0/) transportation focus in the SAR image, With respect to the purpose of segmentation, the aim is to make non-homogeneous areas form a homogenous area in maritime clutter where low wind spiral symptoms, ground belongings, the presentment of ship awakening, etc. are beheld [3]. Clustering is used in [4] to categorize pixels in the image into semantic categories. Segmentation schemes try to identify any background structure or fluctuation.in another hand, object analysis is an efficacious fashion for extracting the dispersion difference of several types of objects and utilize discovering objectives and also ground classifying [5]. Universally, using entropy, information appraises the local distinction among the objective and its abutment [6]. This study reported a ship detection scheme for SAR images using a segmentation-based morphological performance algorithm. The article is ordered as below: In part 2, researches in this field is studied. Part 3 presents the proposed method. In part 4 results in homogeneous, heterogeneous and strong clutter images are presented and finally, the article is summarized in Section 5.

\section{REVIEW ON INFORMATION THEORY AND SEGMENTING}

Vessel tracing utilizing SAR data for ocean management is surveyed during decades. Much research has been done in the forenamed field. Entropy is the evaluation of the amount of information. Object decomposition is an efficacious technique for using the dispersal discrepancy of variant types of objects and using detecting targets and also land subsuming. In [5] relative to the dispersion verification amongst ships and ocean, a novel, disintegration technique by the fame of the Power Entropy (PE) decomposition technique was built. Likewise, two ingredients of dispersion exponent, High-Entropy Scattering Amplitude (HESA) and Low-entropy Scattering Amplitude (LESA) are isolated. Many studies accomplished on the base of that every entropy has been employed to evaluate dissimilarity diversities. Multiscale Variance Weighted Image Entropy (MVWIE) procedure was used to gauge the residential difference amongst object pixels and its neighbors [6]. Dayalan Kasilingam et al. occupied a method entrusted on the minimum entropy filter. It is a filter-based adaptive procedure with minimal adaptive entropy was created to minimize the smearing relic on the moving objects images [7] The Variance Weighted Information Entropy (WIE) and also Joint Radon Transform and Entropy-based detectors were presented in [8] and [9]. The reactance diversity among the vessel and the ocean clutter was analyzed, to determine the ships from the seabed [10]. 
Cross entropy was applied in [11] to evaluate the discrimination among the two forms of scatters, exposing the mean dispersal and finally to get rich witting anent scattering intensity of objects. a further technique called Local contrast variable weighted information entropy (LCVWIE) was stated for intricate backgrounds [12]. A ship tracking system emanates from information theory as well as Harris corner detection is reported in [13]. The Similarity Variance Weighted Information Entropy (SVWIE) was advised to lessen the clutter and enhance the objective [14]. On the other hand a novel method based on the gray-level morphological instauration and the multi-characteristic resolution was introduced in [15] to recognize objects of tiny ships in a heavy maritime background. The suggested technique intercepts the small object from the background ocean clutter. [16] Reports a vessel identification and segmentation system trusted on a modified Mask R-CNN model. A new process to raise ship candidates and recognition based on a modern deep encoding-decoding frame was reported in [17]. Researches utilize a segmentation-based method to trace the vessels. Subsequently, a new system was created to detect the objects in port based on image segmentation for SAR images [18]. [19] Is reporting another algorithm trusted on the Local Binary Pattern (LBP) descriptor and also salience segmentation and composed of the vessel structure. Focused Correlation (FC) method was reported by Alexander Kadyrov et al. to gain robustness to the geometric aberration of content in the image [20]. A ship detection procedure based on morphological component analysis (MCA) is attended from High-Frequency Surface Wave Radar (HFSWR) images [21]. In [22] a method based on Morphological Component Analysis (MCA) that is using High-Frequency Surface Wave (HFSW) radars were studied. Article [23] presents a new cascade-segmentation based and local CFAR detection stage. Subsequently, in the article [24] a method was created to decrease the impact of vessel wakes on the marine recognition procedure by a family segmentation method. The scholars exert a clustering technique rely on density to recognize objects in ocean clutter [25]. Some researchers have applied segmentation for land masking propose. Segmentation can be used on occasions such as the automatic segmentation of the sea area from the ground. However, some deflections that forbid to fragmenting of the ground decently alike few contrasts and severe spots in satellite images. Preprocessing methods such as multi look processing or Kuan and Sigma filters were applied to lessen speckles in the depreciation of spatial resolution [26]. Search for Unidentified Maritime Objects (SUMO) was reported by Harm Greidanus et al. that is working based on the CFAR algorithm for multi-look satellite images [27]. In [28] an unsupervised segmentation method is planned that is relying on a hierarchic triplet Markov field (TMF) within the discrete wavelet amplitude.

\section{PROPOSED METHOD}

Ship identification in heterogeneous, homogeneous and strong clutter SAR images is reported in this paper. Since vessel tracking in heterogeneous background images is difficult due to the tendency of stains and noise, a new method of ship detection based on morphological reconstruction and background and foreground segmentation is offered in this study. In the first step, the gray image is transformed to a binary form with a threshold value (100-180). After that, the noise is removed from the image and then the entropy value is calculated and the entropy filter is applied. In this step, by separating the foreground and background of the image, an attempt is made to identify the candidates that can be identified as ships. Next, at the yield of the pre-processing phase, the morphological verifications are processed on the binarized image to utilize the complexity, shape, and target characteristics of the image. Then, by considering the intensity and specificity of the contrast and target size obtained targets greater than 50 pixels are defined as vessels.

\section{A. Noise removal}

Noise elimination from an image relegates to the signal instauration proceeding of noisy images. Noise harvesting is one of the essential phases of pre-processing. To transmute a gray-scale image to a binary image, it is needed to infix a threshold and put all pixels below the threshold value to 0 and all pixels above the threshold value to 1 (or 255). When analyzing an image, a series of 0 and 1 is attained, which indicates the foreground and background of the image. This binary image can be used to separate an object from the background, amass the pixels of an object, or calculate and extract the characteristic of a target. In this study, it is used for segmentation purposes. Image segmentation is intended to reduce the additional computation and reduce the execution time of the algorithm. In this effort, a trial and error method is selected for threshold value selection for binarization of the image. Twenty images of the homogeneous and heterogeneous scenes were tested and finally determined after reaching a steady-state threshold in the range of 100 to 180 . The reason for using this method is that this range can be applied to all three types of homogeneous, heterogeneous and strong clutter images. The equation (1) is pointing to thresholding relation.

$$
j(x, y)= \begin{cases}b_{0} & i(x, y) \leq t \\ b_{1} & i(x, y)>t\end{cases}
$$

In the above relation, $\mathrm{i}(\mathrm{x}, \mathrm{y})$ is the main image and $\mathrm{j}(\mathrm{x}, \mathrm{y})$ represents the attained binary image.

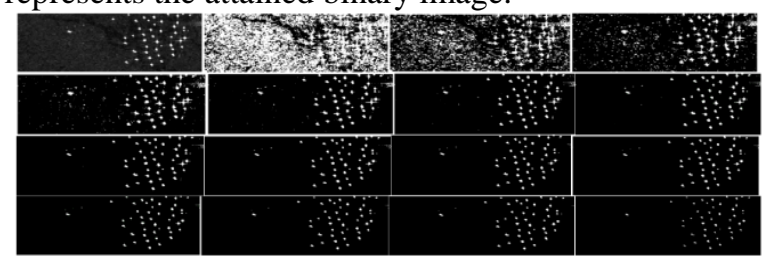

Fig. 1. Threshold value from left to right respectively (image, 30-200)

Figure 1 shows a heterogeneous image with a threshold value of 30 to 200. Thresholding was performed on 20 images, namely 10 homogeneous images and 10 heterogeneous images, respectively. The experiment stopped when it came to a constant suffering. Afterwards, the results showed that the range of 100-180 was appropriate and applicable for all three groups of homogeneous, heterogeneous, and strong clutter SAR images.

Published By: 


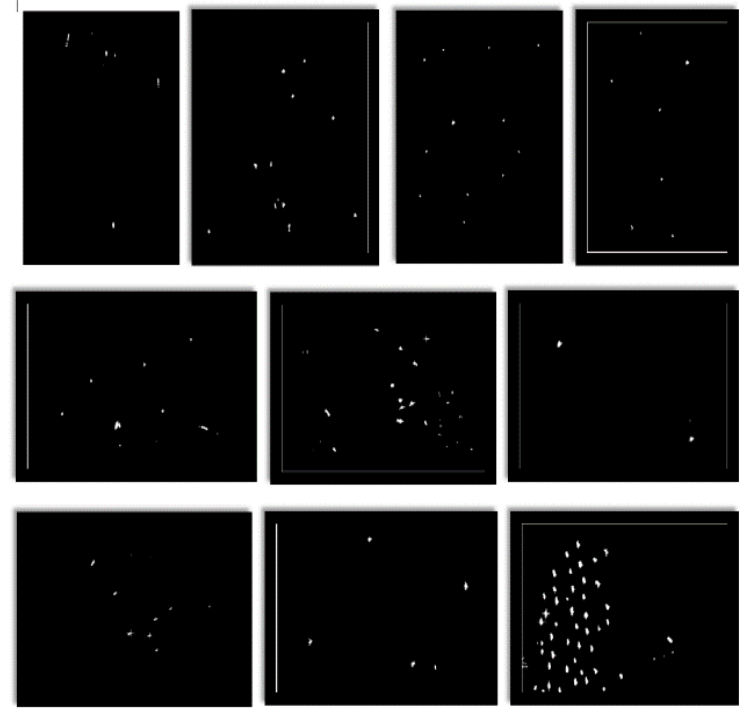

Fig. 2: SAR binarised images with threshold value 120

Figure 2 represents several homogeneous and heterogeneous images typically with a threshold value of 120 to 200.

\section{B.Entropy filtration}

Entropy is the measure of the amount of information and measures the quantity of knowledge. This is a judging of complexity, cohesion, uncertainty [9], and disruption to the random process. Entropy filtering is relying on the displacement of pixel values in the image by entropy value. Entropy is computed in a determined area, commonly from the pixel's n-by-n symmetrical neighbourhood in the input image [29]. The computed entropy is the equation number (2).

$$
F_{(i, j)}=-\sum_{d}^{D} h_{d,(s e)} \log _{2}\left(h_{d,(s e)}\right)
$$

Where se $(i, j)$ the pixel's, $\Phi(i, j)$ neighborhood and $H(d)$ is the normalized histogram function and the below equation should comply below status that is achieving from the description of Shannon entropy as an indicator of information [29] which is shown in relation (3) as below:

$$
S=-\sum_{d}^{D} h_{d} \log _{2}\left(h_{d}\right)
$$

Whereas the output image pertains to the chosen part and the output image may be blurred [29] morphological operators have been utilized to amend the image and vessel identification process. As explained earlier the threshold method is used to convert grayscale images to binary images that are always accompanying errors. The error motive the eventuate noise to be applied to the image. Open and closed morphologies can be employed to clear noise from images [30]. These two operators are exerted altogether with the threshold method. This operator is utilized in structural elements. The threshold section of the algorithm's detection pixels which relieve values over some predesignate threshold accordingly recognize the vessels. Figure 3 demonstrate the flowchart of suggested algorithm.

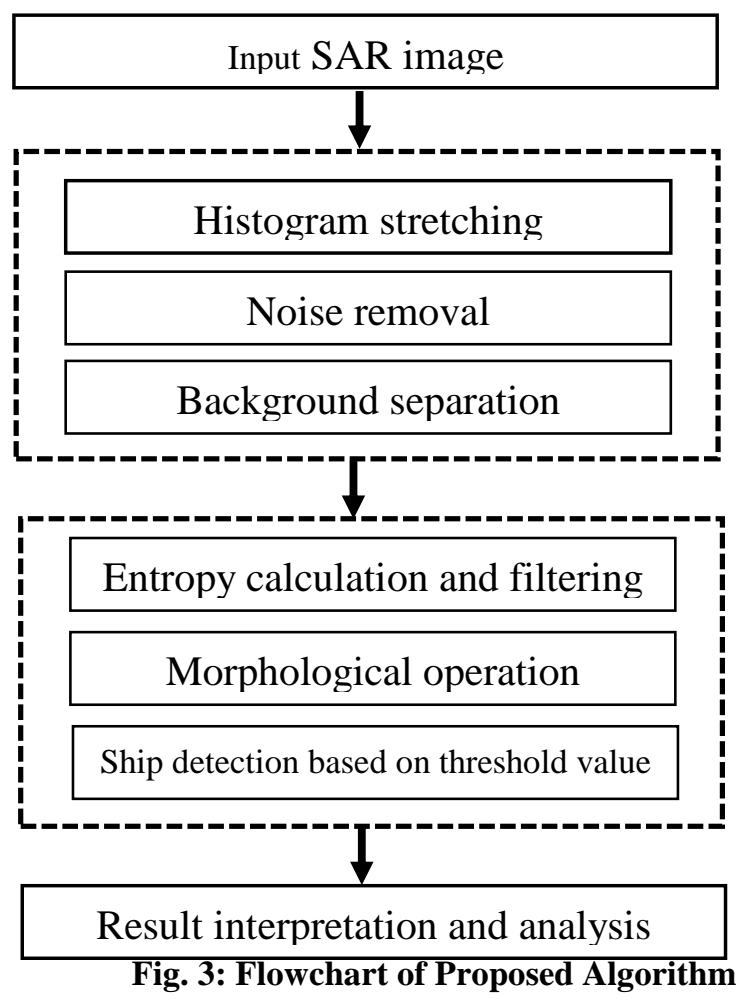

\section{EXPREMENTAL RESULTS AND DISCUSSIONS}

\section{A. Dataset}

In this section, experiments are executed to appraise the proficiency of the suggested method. The sample data set utilized in experimental implementation in this work, taken from Alaska Satellite Facility (ASF) that is making remote-sensing data available. We discovered the targets, using MATLAB and Python software. Figure 4 shows the geographical area used in the study.

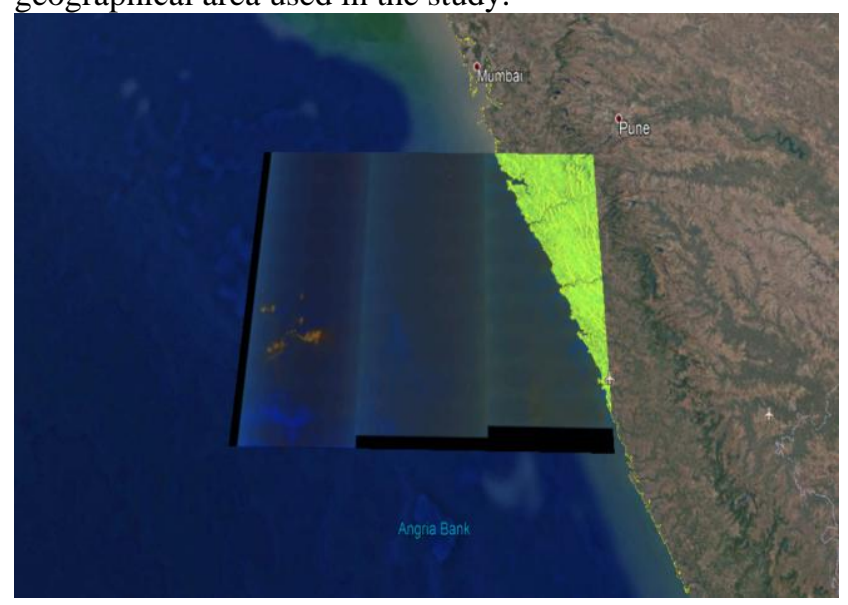

Fig. 4: geographical studied area

\section{B. Detection Results Evaluation}

The figure of merit (FoM) will be calculated as relation (4): $F o M=N_{c d} /\left(N_{f a}+N_{t t}\right)$

$N_{c d}$ : The number of correct detections

$N_{f a}$ : The number of false alarms

$N_{t t}$ : The number of real targets in the original SAR image

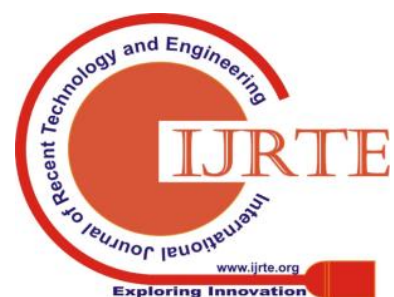




\section{Ship detection based on information theory and segmentation from Synthetic Aperture Radar (SAR) images}

C. Performance on homogenous, heterogeneous and strong clutter scene images

In order to explore the efficiency of the proposed method, homogeneous, heterogeneous, and strong clutter SAR images are tested, respectively. Homogeneous and heterogeneous scenes were taken from a Sentinel-1A SAR image.

- Experiment 1: Ship identification in homogenous image In general, low winds create a clear sea level and poor radar rows that emerge as a homogeneous background in the SAR image. It is generally easier to find the targets in the homogeneous images than heterogeneous images. Figures 5 present the outcome of the proposed method on a homogeneous background. Practical results show promising discovery results.

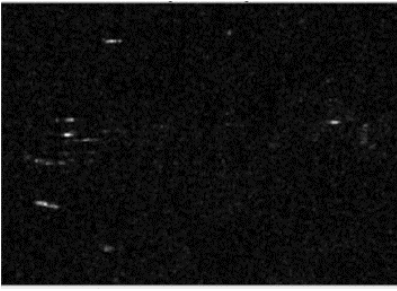

(a)

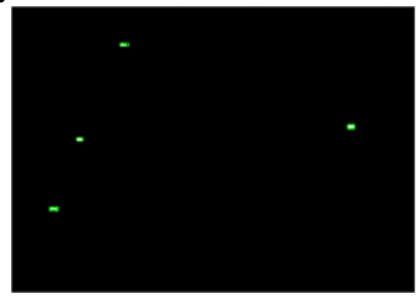

(b)
Fig. 5. Detection results of a homogeneous scene: (a) original SAR image, (b) detection result

- Experiment 2: Ship identification in heterogeneous images

Ship detection in heterogeneous images is more difficult because in heterogeneous sea spike scenes the ship's illumination at sea level may be detected [12]. Figure 6 shows the results of the offered method in a heterogeneous background. (c) Is the original SAR image of a heterogeneous scene containing 45 ships and (d) points to the results of the detection of this method.

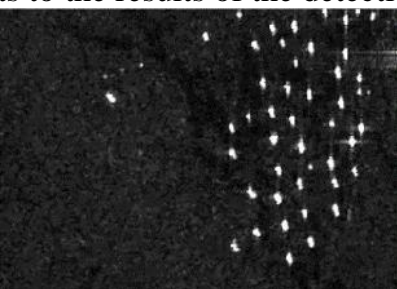

(c)

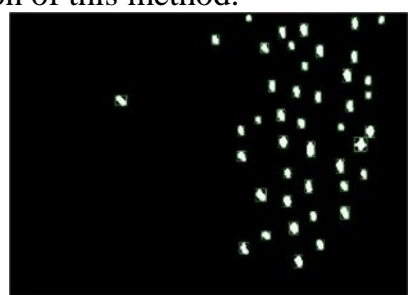

(d)
Fig. 6. Detection results of a heterogeneous scene: (c) original SAR image, (d) detection result

- Experiment 3: Ship identification in Strong clutter image A strong clutter background is a general accident that perceives SAR at angles with high grazing points [12]. Figure 7 demonstrates the results of ship discovery utilizing the proposed method in strong clutter background. The original SAR image of the strong clutter scene is shown in (e) that containing five ships and (f) is the result of proposed method.

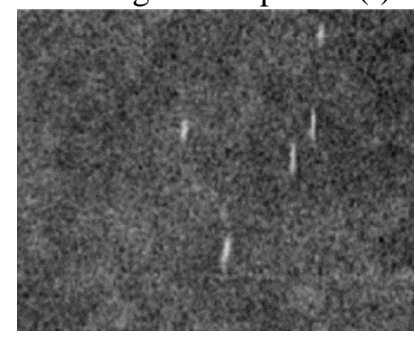

(e)

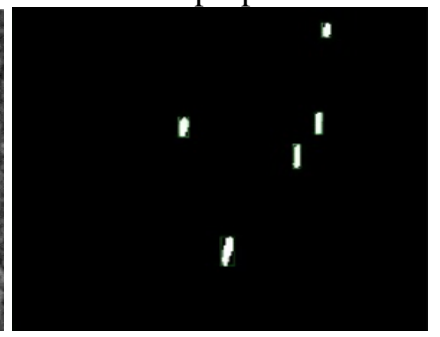

(f)
Fig. 7. Detection results of a strong clutter scene: (e) original SAR image,

(f) detection result
Table I present the outcome of the ship's discovery using the SAR image in the homogeneous, heterogeneous and strong clutter scenes.

Table I: FoMs of the detection results for the three scenes

\begin{tabular}{c|cccc} 
SCENE & $\mathbf{N}_{\mathbf{C D}}$ & $\mathbf{N}_{\mathbf{F A}}$ & $\mathbf{N}_{\text {TT }}$ & $\mathbf{F o M}$ \\
\hline Homogeneous & 5 & 0 & 5 & 1 \\
Heterogeneous & 43 & 2 & 45 & 0.91 \\
Strong Clutter & 5 & 0 & 5 & 1
\end{tabular}

The reported method was compared with the Local Contrast Variance Weighted Information Entropy (LCVWIE) method [12] and Polarimetric Covariance Difference Matrix PCDM-Shannon entropy based method [31] algorithms. The results indicates that the algorithm mentioned in the heterogeneous images and the strong clutter images are better and equal according to the PCDM-Shannon entropy method and the LCVWIE methods. The result of FoM showed in tables II and III for LCVWIE method and PCDM-Shannon entropy based method respectively. The results shows that the identified ships using desired method are equal to their competitors.

Table II: FoM result of proposed method compared with LCVWIE method

\begin{tabular}{l|cccc} 
METHOD NAME & NCD & NFA & NTT & FOM \\
\hline LCVWIE Method & 4 & 0 & 4 & 1 \\
Proposed Method & 4 & 0 & 4 & 1
\end{tabular}

Table III. FoM result of proposed method compared with PCDM-Shannon entropy Method

\begin{tabular}{c|cccc} 
METHOD NAME & $\mathbf{N}_{\text {CD }}$ & $\mathbf{N}_{\mathbf{F A}}$ & $\mathbf{N}_{\text {TT }}$ & FOM \\
\hline $\begin{array}{c}\text { PCDM-Shannon } \\
\text { Entropy Based Method }\end{array}$ & 5 & 0 & 5 & 1 \\
$\begin{array}{c}\text { Proposed Method } \\
\text { Propos }\end{array}$ & 5 & 0 & 5 & 1
\end{tabular}

\section{CONCLUSION}

In this article, an entropy and morphological operations based algorithm for ship detection form, SAR images were presented. In the first step, the noise removed from the gray image and becomes a binary image. Subsequently, entropy filter operation and morphological operators were applied to detect the ship with respect to a predesignate threshold, respectively. Experiments on homogenous, heterogeneous and strong clutter SAR images showed that using entropy and segmentation based on morphological operation can improve the vessel identification process. Finally, as the algorithm used a threshold in a range for noise removing, so part of the future work is automatic threshold adjustment.

\section{REFERENCES}

1. N. Seltenrich, "REMOTE-SENSING APPLICATIONS for ENVIRONMENTAL HEALTH RESEARCH." Environmental Health Perspectives, vol. 122, no. 10, pp. 268-A275, 2014.

2. J. Cicuendez Perez, M. Alvarez Alvarez, J. Heikkonen, J. Guillen and T. Barbas, "The Efficiency of Using Remote Sensing for Fisheries Enforcement: Application to the Mediterranean Bluefin tuna fishery," Elsevier B.V. Fisheries Research, vol. 147, pp. 24-31, 2013.

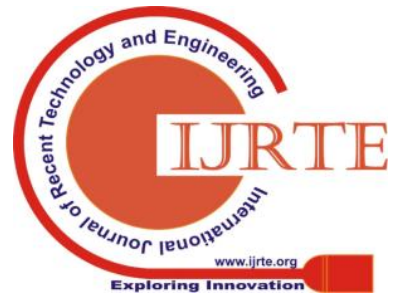


3. P. L. Massimo Sciotti, "Ship Detection in SAR Images: a Segmentation-Based Approach," in Proceedings of the 2001 IEEE Radar Conference, Piscataway, NJ, USA, 2001.

4. W. B. G. R. B. J. S. T. W. a. T. S. CScott Brown, "Image Segmentation Stability: An Empirical Investigation," in SoutheastCon 2018, St. Petersburg, FL, USA, 2018.

5. S. G. J. H. G. L. Gui Gao, "Adaptive Ship Detection in Hybrid-Polarimetric SAR Images Based on the Power-Entropy Decomposition," IEEE TRANSACTIONS ON GEOSCIENCE AND REMOTE SENSING, vol. 56, no. 9, pp. 5394-5407, 2018.

6. X. W. a. C. Chen, "Ship Detection for Complex Background SAR Images Based on a Multiscale Variance Weighted Image Entropy Method," IEEE GEOSCIENCE AND REMOTE SENSING LETTERS, vol. 14, no. 2, pp. 184-187, 2017.

7. J . W. J.-S. L. a. R. J. Dayalan Kasilingam, "Focusing of Synthetic Aperture Radar Images of Moving Targets using Minimum Entropy Adaptive Filters," in IEEE International Geoscience and Remote Sensing Symposium. Taking the Pulse of the Planet: The Role of Remote Sensing in Managing the Environment. , Honolulu, HI, USA, USA, 2000.

8. Y. Z. J. Y. a. L. C. L. Yang, "Variance WIE based infrared images processing," IET Electronics Letters, vol. 42, no. 15, pp. 857 - 859, 2006.

9. Z. T. Y. W. Y. S. Pengfei Du, "Ship Wakes Detection in SAR Images Based on the Joint Radon Transform and Entropy," in IEEE, CIE International Conference on Radar Radar, 2006. CIE '06. International Conference, Shanghai, China, 2006.

10. Z. Z., B. W. L. L. a. L. M. Xiaowu Xiao, "Ship Detection under Complex Backgrounds Based on Accurate Rotated Anchor Boxes from Paired Semantic Segmentation," MDPI, vol. 11, no. 2506, pp. 1-18, 2019.

11. J. Y. Y. LishengFan, "A cross-entropy based parameter for ship detection from a polarimetric SAR image," in IEEE International Symposium on Microwave, Antenna, Propagation and EMC Technologies for Wireless Communications Proceedings, Piscataway, NJ, USA, 2005.

12. Y. H. J. P. Q. Z. Q. G. a. J. Y. Weibo Huo, "Ship Detection from Ocean SAR Image Based on Local Contrast Variance Weighted Information Entropy," MDPI, vol. 18, no. 1196, pp. 1-15, 2018.

13. H. W. S. L. M. S. a. X. L. Yangyang Deng, "Analysis of the ship target detection in high-resolution SAR images based on information theory and Harris corner detectio," EURASIP Journal on Wireless Communications and Networking, vol. 2018, no. 1, pp. 1-9, 2018.

14. Y.-H. Z. a. P. Liu, "Adaptive Ship Detection for Single-Look Complex SAR Images Based on SVWIE-Noncircularity Decomposition," MDPI, vol. 18, no. 3293, pp. 1-15, 2018.

15. Z. L., Y. Z., B. L., W. X. a. Y. H. Yongsong Li, "Thermal Infrared Small Ship Detection in Sea Clutter Based on Morphological Reconstruction and Multi-Feature Analysis," MDPI, vol. 9, no. 3786, p. 29, 2019.

16. M. D., H. D., B. H., A. E. K. W. XUAN NIE, "Attention Mask R-CNN for Ship Detection and Segmentation From Remote Sensing Images," IEEE Access, vol. 8, no. 2020, pp. 9325-9334, 2020.

17. X. Xiao, Z. Zhou, B. Wang and Z. An, "Accurate Ship Detection via Paired Semantic Segmentation," in Chinese Control and Decision Conference (CCDC) Control and Decision Conference (CCDC), Nanchang, China, 2019.

18. L. Zhai, Y. Li and Y. Su, "Segmentation-based ship detection in harbor for SAR images," in CIE International Conference on Radar, RADAR, 2017.

19. F. Yang, Q. Xu and B. Li, " Ship Detection From Optical Satellite Images Based on Saliency Segmentation and Structure-LBP Feature," Remote Sensing Lett. Geoscience and Remote Sensing Letters, IEEE., pp. 602-606, May 2017.

20. H. Y. a. H. L. Alexander Kadyrov, "Ship Detection and Segmentation using Image Correlation," in IEEE International Conference on Systems, Man, and Cybernetics, Manchester, UK, 2013.

21. S. Grosdidier and A. Baussard, "Ship detection based on morphological component analysis of high-frequency surface wave radar images.," IET Radar, Sonar \& Navigation, vol. 6, no. 9, pp. 813-821, 2012

22. A. B. a. A. K. Samuel Grosdidier, "Morphological-based source extraction method for HFSW radar ship detection," in IEEE International Geoscience and Remote Sensing Symposium Geoscience and Remote Sensing Symposium (IGARSS), Honolulu, HI, USA, 2010.

23. G. S. G. L. J. C. Gui Gao, "Performance Comparison Between Reflection Symmetry Metric and Product of Multilook Amplitudes for
Ship Detection in Dual-Polarization SAR Images," IEEE JOURNAL OF SELECTED TOPICS IN APPLIED EARTH OBSERVATIONS AND REMOTE SENSING, vol. 10, no. 11, pp. 5026-5038, 2017.

24. W. Q. Xia, "Moving Target Detection for THz SAR Systems based on Multilook Processing," in IEEE Asia-Pacific Conference on Antennas and Propagation (APCAP) Antennas and Propagation (APCAP), Auckland, New Zealand, 2018.

25. Y. X. X. Z. Haitao Lang, "Ship Detection in High-Resolution SAR Images by Clustering Spatially Enhanced Pixel Descripto," IEEE Transactions on Geoscience and Remote Sensing, vol. 57, no. 8, pp. 5407 - 5423, 2019.

26. X. X. Dandan Gu, "A Novel Procedure for Land Masking in Ocean-Land Segmentation from SAR Images," in IEEE International Conference on Signal Processing, Communications and Computing (ICSPCC) Signal Processing, Communications and Computing (ICSPCC), Beijing, China, 2014.

27. M. A. C. S. F.-X. T. N. K. ,. A. Harm Greidanus, "The SUMO Ship Detector Algorithm for Satellite Radar Images," MDPI AG, vol. 9, no. 3, pp. 1-27, 2017.

28. S. L. Z. JiajingWang, " Unsupervised SAR Image Segmentation Based on a Hierarchical TMF Model in the Discrete Wavelet Domain for Sea Area Detection," Discrete Dynamics in Nature and Society, vol. 2014 no. 2014, pp. 1-10, 2014.

29. J. Urban, "Information Entropy," in Applications from Engineering with MATLAB Concepts, intechopen, 2016, pp. 43-71.

30. I. K. Igor VUJOVIĆ, "Oil Spills Detection from SAR Images Using Wavelets," Turkish Journal of Maritime and Marine Sciences , vol. 4, no. 1, pp. 73-80, 2018.

31. H. Z. C. W. Q. F. a. L. X. Cheng Zhang, "A Novel Ship Detection Method Based on Shannon Entropy in Chinese Gaofen-3 Fully Polarimetric SAR Images," in Progress In Electromagnetics Research Symposium, Singapore, 2017.

\section{AUTHORS PROFILE}

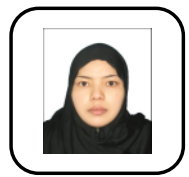

Somaya Jafari doing M.tech in Computer Science and Engineering, Vellore Institute of Technology, Vellore, Tamilnadu, India. The interest fields of research is remote sensing applications, image processing and also computer vision.

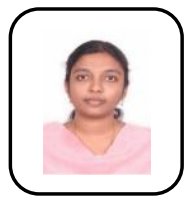

Dr. Anisha M. Lal is an Associate Professor in the School of Computer Science and Engineering at Vellore Institute of Technology, Vellore, India. She received her B.E and M.E degrees in Computer Science and Engineering from Anna University in 2003 and 2005, respectively. She has been awarded her Doctorate in Computer Science and Engineering at Vellore Institute of Technology, Vellore in 2016. She has teaching and research experience of about 14 years. She has published more than 20 papers in international journals and presented around 4 papers in national and international conferences. Her research interests include Image Processing, Remote Sensing, Biomedical, Big Data and Soft Computing. She has served as a reviewer in many academic conferences and journals in the related areas.

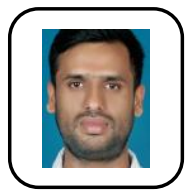

Jagalingam Pushparaj is a senior assistant professor at Vellore institute of technology (VIT), Vellore, India. He received his Bachelor of Engineering Degree in Computer Science and Engineering from Anna University, India. His Masters was in Remote Sensing and GIS from Adhiyamaan Engineering College, Hosur, India. He received the doctoral degree from National Institute of Technology Karnataka, Surathkal, India. His research interests are image processing, deep learning, ocean studies and Remote Sensing \& GIS applications. 